\title{
Two faces of bivalent domain regulate VEGFA responsiveness and angiogenesis
}

\author{
Jiahuan Chen ${ }^{1}$, Xiaodong Liang1', Shasha Zhang ${ }^{1}$, Shiyan Wang ${ }^{1,2}$, Sara P. Garcia ${ }^{3}$, Pengyi Yan ${ }^{1}$, Huijing Yu', Zixuan Li', \\ Li Liu', Fang Zhang', Weiting Wei ${ }^{1}$, Huangying Le', Yan Zhang ${ }^{4}$, Guo-cheng Yuan $\mathbb{0}^{3}$, Sun Chen ${ }^{1}$, Yingwei Chen', \\ Kun Sun', William T. Pu $\mathbb{1}^{5,6}$ and Bing Zhang ${ }^{1}$
}

\begin{abstract}
The bivalent domain $(\mathrm{BD})$ at promoter region is an unique epigenetic feature poised for activation or repression during cell differentiation in embryonic stem cell. However, the function of BDs in already differentiated cells remains exclusive. By profiling the epigenetic landscape of endothelial cells during VEGFA (vascular endothelial growth factor A) stimulation, we discovered that BDs are widespread in endothelial cells and preferentially marked genes responsive to VEGFA. The BDs responsive to VEGFA have more permissive chromatin environment comparing to other BDs. The initial activation of bivalent genes depends on RNAPII pausing release induced by EZH1 rather than removal of H3K27me3. The later suppression of bivalent gene expression depended on KDM5A recruitment by its interaction with PRC2. Importantly, EZH1 promoted both in vitro and in vivo angiogenesis by upregulating EGR3, whereas KDM5A dampened angiogenesis. Collectively, this study demonstrates a novel dual function of BDs in endothelial cells to control VEGF responsiveness and angiogenesis.
\end{abstract}

\section{Introduction}

In the past 30 years, histone modifications and chromatin-modifying enzymes emerged as essential regulators of gene expression ${ }^{1}$. Most functional genomic regions featured with covalent histone modifications link to gene activation ( $\mathrm{H} 3 \mathrm{~K} 4 \mathrm{me} 3$ and $\mathrm{H} 3 \mathrm{~K} 27 \mathrm{ac}$ ) or repression (H3K27me3 and H3K9me3) $)^{1,2}$. In 2006, Bernstein et al. ${ }^{3}$ analyzed the chromatin occupancy of a series of histone modifications in mouse embryonic stem (ES) cells and found that $75 \%$ of $\mathrm{H} 3 \mathrm{~K} 27 \mathrm{me} 3$ promoter regions were also marked by H3K4me3. This class of promoter regions with both activating H3K4me3 and repressive H3K27me3 marks was named the bivalent domain (BD). Subsequent

\footnotetext{
Correspondence: Bing Zhang (bingzhang@sjtu.edu.cn)

'Department of Pediatric Cardiology, Xin Hua Hospital, School of Medicine, Key Laboratory of Systems Biomedicine, Shanghai Center for Systems Biomedicine, Shanghai Jiao Tong University, Shanghai 200240, China

${ }^{2}$ School of Life Science and Food Engineering, Huaiyin Institute of Technology, Huaian 223003, China

Full list of author information is available at the end of the article. These authors contributed equally: Jiahuan Chen, Xiaodong Liang Edited by R. Mantovani
}

studies showed that BDs were found widely in tissues of higher vertebrates, such as zebrafish and mammals, but not in lower vertebrates and arthropods, such as Xenopus and Drosophila ${ }^{4}$.

In ES cells, bivalent genes are enriched for developmental transcription factors and expressed at low levels. Upon lineage-specific differentiation, some bivalent genes lost H3K27me3 and became fully activated, whereas others lost H3K4me3 and were silenced ${ }^{3}$. These observations suggested that BDs in ES cell provide a permissive environment for developmental genes to turn on or off in response to developmental cues. In mature, differentiated tissues, BDs are also present, but their function is not well understood. Are they merely leftover from cell commitment, or do they possess other important functions?

The generation of BDs requires both Trithorax and Polycomb complexes. H3K4me3 of BDs is established by two types of Trithorax methyltransferases, MLL and SET1A/B, which are recruited to CpG islands by CXXC domain-containing proteins ${ }^{5}$. H3K4me3 demethylases of the Jarid/KDM5 classes, including KDM5A (Jarid1a) and 
KDM5B (Jarid1b), compete with the methyltransferases and erase H3K4me3 (ref. ${ }^{6}$ ). On the other hand, the repressive mark of H3K27me3 is created and maintained by Polycomb complexes, including Polycomb complex 1 (PRC1) and Polycomb complex 2 (PRC2). The methyltransferase subunit of PRC2 is either EZH1 or EZH2 (ref. ${ }^{7}$ ). Removal of H3K27me3 at BDs requires the demethylase UTX, a component of MLL3/4 complexes. Intriguingly, PRC2 is able to recruit KDM5A to $\mathrm{BD}$ and mediate the demethylation of H3K4me3, illustrating a shared machinery between active and repressive mark. Loss or gain of either H3K27me3 or H3K4me3 is a major mechanism reported previously for activating or silencing the transcription of bivalent genes ${ }^{8,9}$. However, it was still unclear if the BD and its catalytic machineries involve in the regulation of transcription response to environmental stress, such as VEGFA (vascular endothelial growth factor A) stimulation.

VEGFA governs vasculogenesis and angiogenesis. AntiVEGFA therapy emerged as an important approach to treat certain cancers and age-related macular degeneration ${ }^{10}$. VEGFA interacts with VEGFA receptors, mainly VEGFA receptor 2 in endothelial cells, to promote endothelial cell proliferation, migration, and permeability. We and others showed that VEGFA induces rapid and pervasive gene expression changes in endothelial cells. For instance, our recent study showed that VEGFA promoted gene expression and angiogenesis by stimulating acetylation of ETS1 or increasing genome-wide deposition of H3K27ac. Despite these advances, many gaps remain in the understanding of how VEGFA modulates the epigenome and regulates gene transcription.

\section{Results}

\section{BD associated with VEGFA-responsive genes}

Our model system consisted of human umbilical vein endothelial cells (HUVECs) that were cultured overnight in low serum and no VEGFA, and then stimulated for $12 \mathrm{~h}$ with $50 \mathrm{ng} / \mathrm{ml}$ VEGFA. Samples were collected at 0 (unstimulated), 1, 4, and $12 \mathrm{~h}$. To interrogate if the integrated pattern of histone modifications configured chromatin responsiveness to VEGFA, we used chromatin immunoprecipitation followed by high-throughput sequencing (ChIP-seq) to profile the genomic occupancy of multiple histone modifications, including H3K4me2 (a mark of both active promoters and enhancers), H3K4me3 (a mark of active promoters), H3K27ac (a mark of active enhancers and promoters), and H3K27me3 (a mark of repressed enhancers and promoters). Using our previously reported RNA sequencing (RNA-seq) expression profiles for this experimental system ${ }^{11}$, we identified 29,722 expressed genes (RPKM $\geq 1$ at any of the four tested time points). At the promoters of these genes (TSS $\pm 1 \mathrm{~kb}$ ), the ChIP-seq signals of these four histone modifications were grouped into six clusters using $K$-means clustering and the optimal $K$ was determined by Silhouette algorithm (Methods section). Each cluster had $>1000$ genes (Fig. 1a, Supplementary Table 1). Cluster $\mathrm{C} 1$ did not have significant signal at the promoter for any of the profiled histone modifications. C2 had weak H3K27ac but no other active histone marks. C3-6 had at least two active histone marks present. Among them, C5, containing high signals for all three activating histone marks, was the most dominant cluster (13,276 TSSes). Importantly, there were a total of 3379 promoters in C3 and C6 that were occupied by both H3K4me3 and H3K27me3, suggesting they were BDs (Fig. 1a, Supplementary Table 1).

In the same model system, our previous studies identified 901 genes that were significantly changed by VEGFA (differentially expressed genes: DEG, Supplementary Table 2). To further probe the association of these epigenetic patterns with DEG at transcriptional start site (TSS), we calculated the number and enrichment score of DEG in each histone modification cluster (Fisher's exact test; see Methods section; Fig. 1a, right side). DEGs were most highly enriched in C5, the cluster with strong signals for all of the active histone marks. Surprisingly, C3 and C6, which had both active and repressive marks, were also enriched with DEGs $\left(P=1.0009 \times 10^{-11}\right.$ and $1.935 \times 10^{-16}$, respectively, Fisher's exact test). This suggested a possibility that the bivalent epigenetic signature contributed to the responsiveness of VEGFA. To further test this hypothesis, we used a more pervasive approach based on the co-occurrence of H3K27me3 and H3K4me3 at the same promoter to identify BD promoters. This identified 1851 BD promoters (Supplementary Table 1). Histone tag heatmaps at the promoters showed co-occurrences of both H3K4me3 and H3K27me3 (Fig. 1b), and $81.79 \%$ of these promoters overlapped with C3 and C6 (Supplementary Fig. 1a). The expression level of bivalent genes was lower than all expressed genes (BD vs all expressed genes, $P<2.2 \times$ $10^{-16}$, Wilcoxon rank-sum test, Supplementary Fig. 1b), consistent with observations in other cell lineages ${ }^{3}$. When considering TSSs targeted by H3K4me3 with or without H3K27me3, genes with only H3K4me3 showed significantly higher fragments per kilobase of exon model per million reads mapped (FPKM) than bivalent DEGs (bdDEG; $P=0.007671)$ and BDs $\left(P<2.2 \times 10^{-16}\right.$; Supplementary Fig. 1c). Therefore, we used these 1851 peakoverlapping $\mathrm{BD}$ promoters for the following analysis.

We observed that there were more DEGs with promoter BDs than expected by random chance $(69 / 901 ; P<0.001$, Chi-square test; Fig. 1c, Supplementary Fig. 1c). Some bdDEGs had key functions in vascular identity and homeostasis (representative genes are listed on the right side of Fig. 1d). For instance, the Notch pathway ligand DLL4 antagonizes VEGF's proangiogenic effect and promotes tip cells to stalk cells transition ${ }^{12}$. FOXC1 is an 


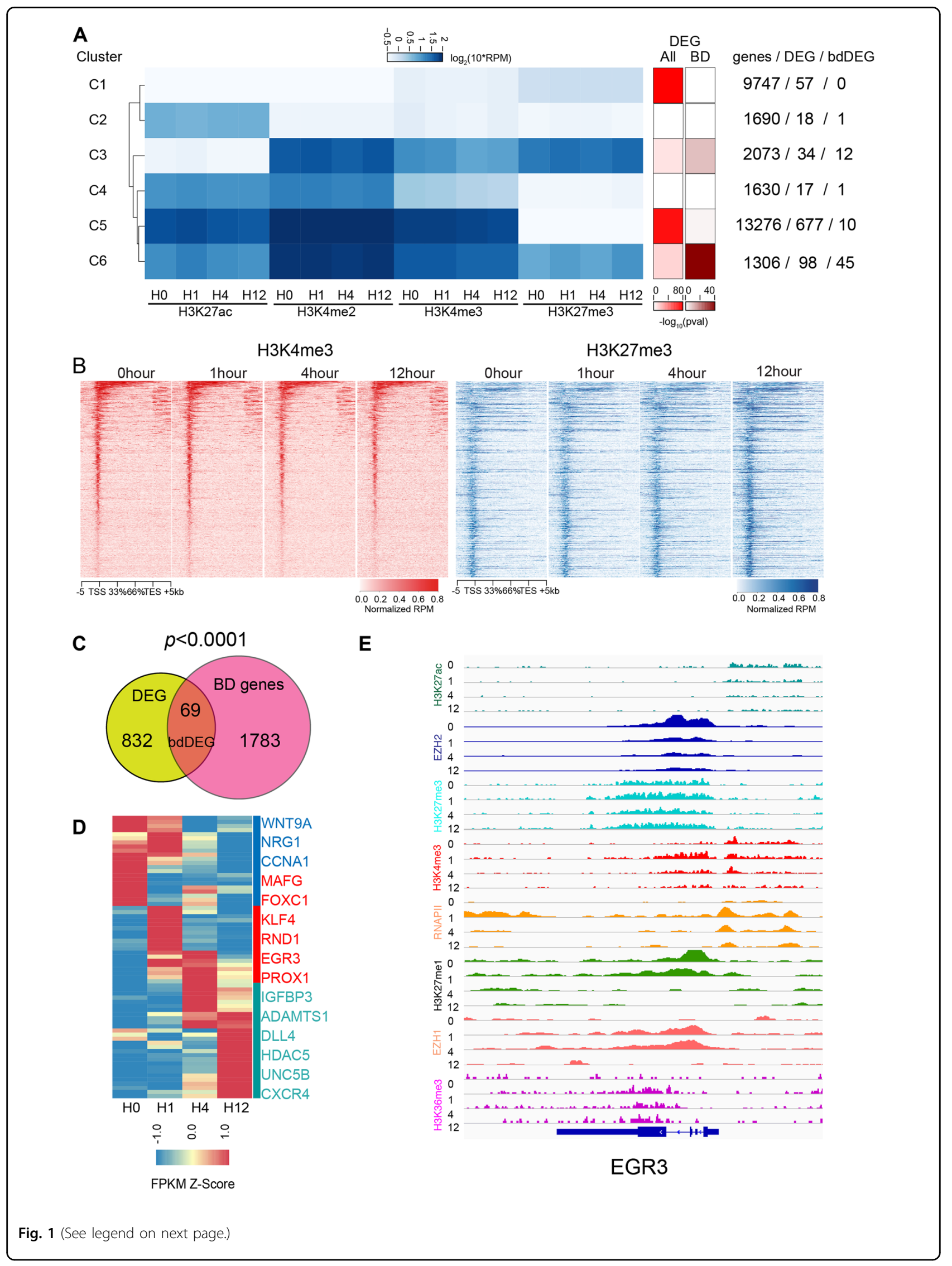


(see figure on previous page)

Fig. 1 DEGs were enriched at BDs. a The correlation between histone clusters and DEG. The left heatmap is the K-means clusters of four different histone marks (H3K4me3, H3K4me2, H3K27ac, and H3K27me3). The middle and the right heatmaps represent the enrichment of DEGs in the set of all genes and BD genes, respectively, at divergent histone clusters by Fisher's exact test. The text on the right side described the number of TSS, DEG, and bdDEG within each cluster. b Tag heatmaps of H3K4me3 and H3K27me3 at all BDs called by H3K27me3 and H3K4me3 peak overlap. c Venn diagram showing the overlap between DEGs and BD genes. We found 69 DEGs were marked by BD, a highly significant enrichment compared to expectations of randomness. Chi-squared test: $P<0.05$ indicated significance. $\mathbf{d}$ Heatmap of bdDEGs from RNA-seq, grouped into three clusters: downregulated genes (blue), early-upregulated genes (red), and late-upregulated genes (jasper). Representative genes within each cluster are listed on the right. e Snapshot of Integrative Genomics Viewer of histone marks, RNAPII, and transcribed RNA near EGR3, a bdDEG that was rapidly and transiently upregulated by VEGFA.

upstream regulator of $D L L 4^{13}$. Both DLL4 and FOXC1 have bivalent promoters, suggesting that bivalency may play an essential role in NOTCH signaling (Fig. 1d). A subset of endothelial cell transcription factors also had bivalent promoters, including $K L F 4$, a master regulator of endothelial cell identity and hemodynamics ${ }^{14}$ (Fig. 1d) and EGR3, which has multiple functions in endothelial cell growth, sprouting, and tube formation and was upregulated $>100$-fold after $1 \mathrm{~h}$ of VEGFA stimulation (Fig. 1d, e). Together, enrichment of BDs at the promoters of DEGs and the key functions of bdDEGs in endothelial biology indicated that $\mathrm{BD}$ may have an irreplaceable role in mediating the proangiogenic effect of the VEGFA pathway.

\section{Epigenetic signature of bdDEG}

To further understand the mechanism underlying alternative expression of bdDEG, we compared the epigenetic features of up- and downregulated bdDEGs to stable BD genes that were not changed by VEGF (Fig. 2a). bdDEG (middle and right columns) showed stronger H3K4me3 and weaker H3K27me3 at their TSSs comparing to stable BD genes (left column). Further separating the upregulated bdDEG to early (gene upregulated at $1 \mathrm{~h}$ ) and late upregulation (gene upregulated at 4-12 h) group demonstrated a significant and early induction of H3K4me3 and H3K27me3 in early but not in lateupregulated bdDEG group (Supplementary Fig. 2). Upregulated bdDEG also had stronger H3K36me3, a mark of active mRNA transcription, at their gene bodies (Fig. 2a). Surprisingly, H3K27ac, a canonical active histone modification on the same lysine residual of histone H3 with H3K27me3, was present at bdDEGs with remarkably higher signals than at non-bdDEGs. H3K27ac had very little overlap with H3K27me3, which was consistent with the fact that they are mutually exclusive in biochemistry (Fig. 2a). Both up- and downregulated bdDEG had elevated H3K27ac, but the pattern was different, with upregulated bdDEGs having a sharper H3K27ac peak near the TSS and overlapping with H3K4me3, and downregulated bdDEG having a more diffusive H3K27ac peak across up- and downstream of TSS (Fig. 2a). H3K27ac upregulation at $1 \mathrm{~h}$ after VEGFA treatment was mainly contributed by early upregulated
bdDEGs (Supplementary Fig. 2). In line with the presence of multiple active histone marks at BD, H3K27me1 with a recently suggested role in positively regulating transcription, also weakly presented at all BD genes (Fig. 2a) ${ }^{15,16}$. Early compared to late-upregulated bdDEGs demonstrated more dramatic augment in H3K27me1 deposition (Supplementary Fig. 2).

Of note, promoter occupancy by EZH2, the methyltransferase catalytic subunit of PRC2, markedly decreased after VEGFA treatment in BD genes (Fig. 2a). PRC1, the first discovered Polycomb complex, at many loci, was found to cooperate with PRC2 in setting up the $\mathrm{BD}^{4}$. To determine if PRC1 has a similar function to maintain the bivalency in the endothelial cells as well, we measured the chromatin occupancy of RINGB, a catalytic subunit of PRC1 that ubiquitinates H2AK119 to deepen the transcription repression ${ }^{17,18}$. Less RINGB occupancy at bdDEG (genes with blue bars) was observed compared to that at unresponsive BD (genes with carmine bars, Fig. 2b). Together, these results suggest that bdDEGs had a more permissive chromatin state for transcription manifested by higher occupancy of active histone marks (H3K27ac, H3K36me3, andH3K4me3) and less occupancy of repressive marks (H3K27me3 and PRC1) compared to other BD genes that were not differentially expressed.

\section{EZH1 mediated the activation of bivalent genes}

Opposite to the primary role of $\mathrm{BD}$ in priming gene expression initially discovered in ES cells ${ }^{3}$, there are 45 genes among bdDEG were upregulated within $12 \mathrm{~h}$ course of VEGFA stimulation. In the following study, we set out to uncover this intrigue mechanism underlying the activation of these genes. Loss of the H3K27me3 repressive mark at bivalent genes was identified as a mechanism activating the BD-marked genes during the commitment of ES cell to tissue lineage. However, our ChIP-seq results showed that H3K27me3 mildly increased rather decreased at activated bdDEGs (middle panel, Fig. 2a). The chromatin signal of UTX and JMJD3, two demethylases specifically in charge with H3K27me3 demethylation, either maintained or reduced their chromatin occupancy at most bdDEG loci (Fig. 3a, b). These data suggested that VEGFA-dependent 

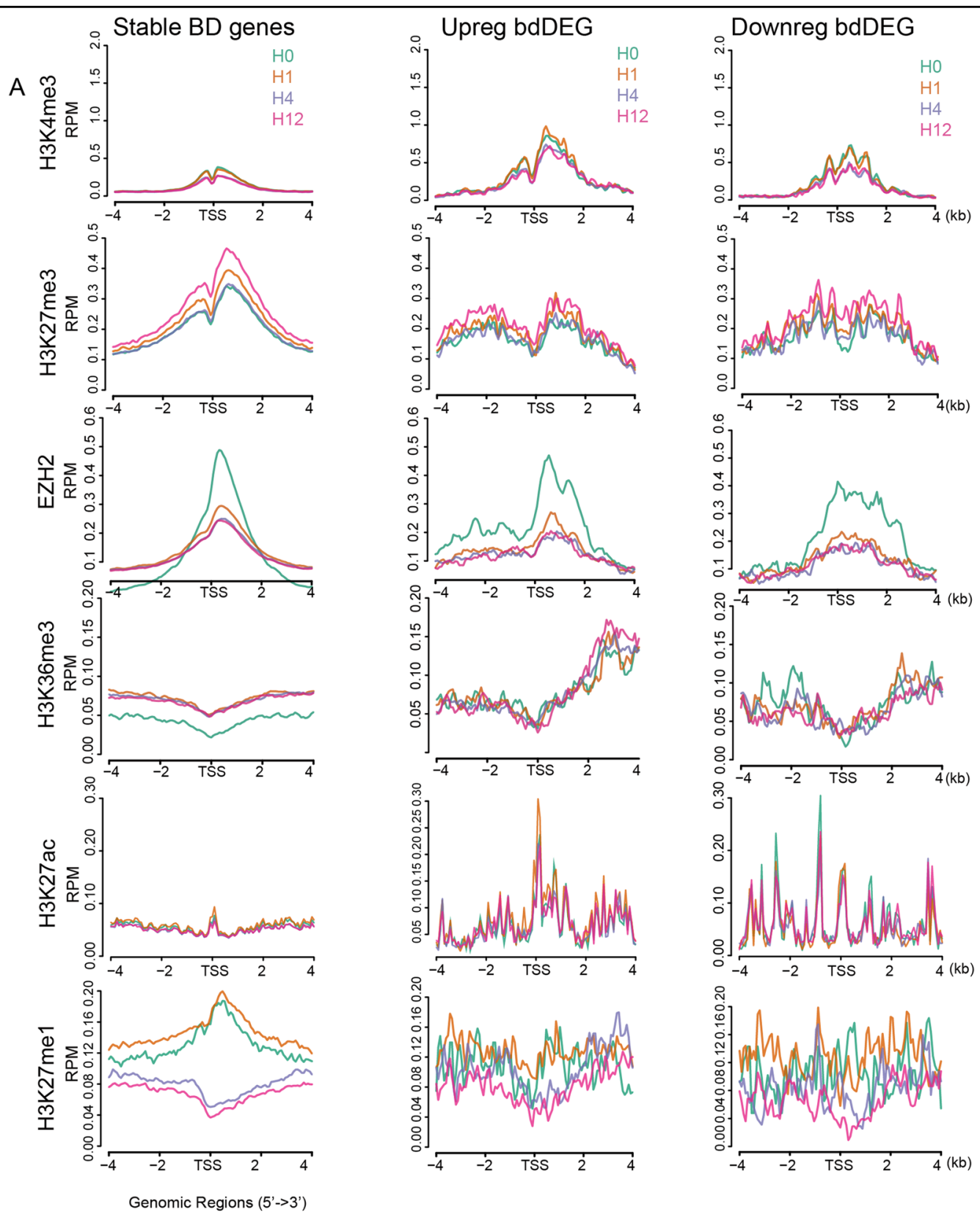

B

RINGB
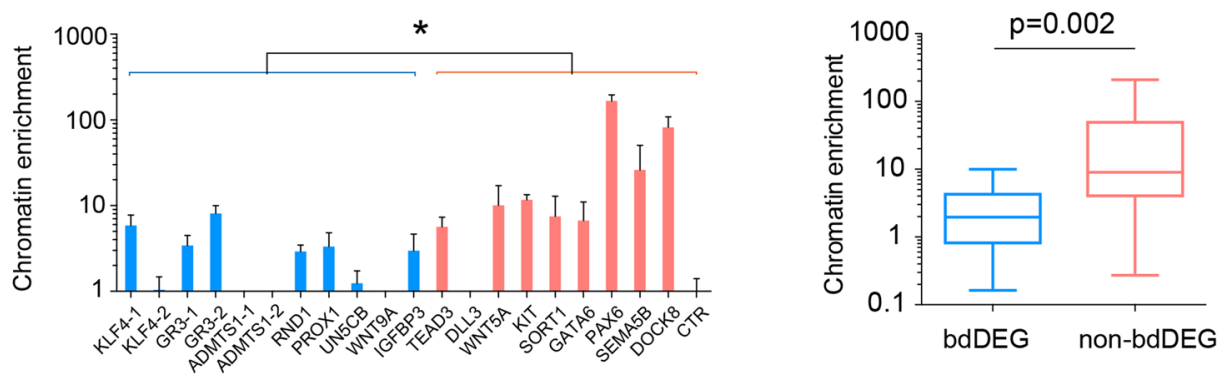

Fig. 2 Chromatin features of bdDEG at TSS. a Aggregation plots of histone modifications near promoters of all BD genes (left) and upregulated (middle) or downregulated (right) bdDEGs. b RINGB chromatin occupancy at BD genes that were (bdDEG, blue) or were not (non-bdDEG, megenta) differentially expressed, as measured by ChIP-qPCR. Box plot on the right summarizes the chromatin enrichment of RINGB at each class of genes; $n=$ 4 for each gene loci, Mann-Whitney $U$ test. 


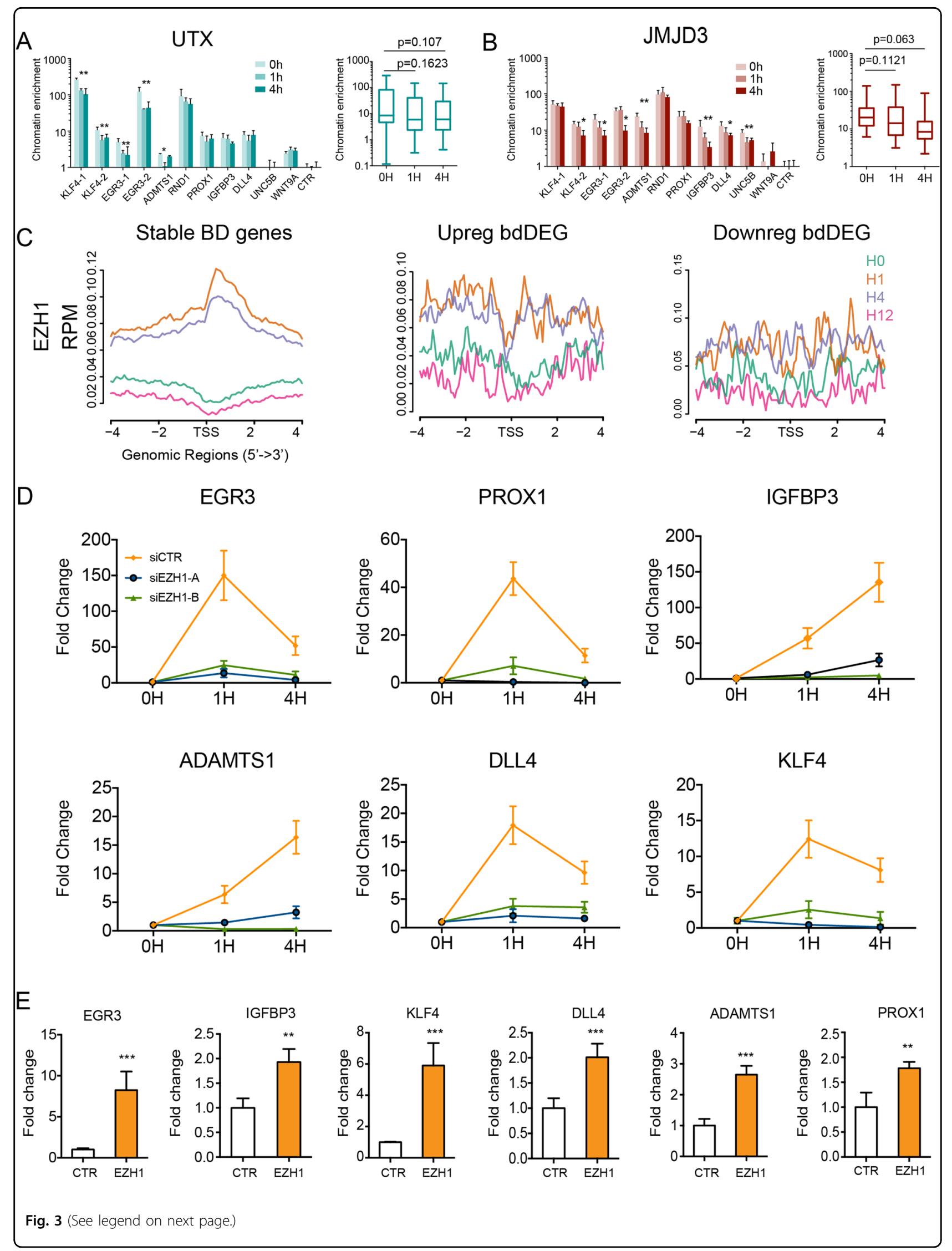


(see figure on previous page)

Fig. 3 VEGFA treatment increased the occupancy of EZH1 complex at upregulated bdDEG. $\mathbf{a}, \mathbf{b}$ UTX $\mathbf{a}$ and JMJD3 $\mathbf{b}$ chromatin occupancy at bdDEG, as measured by ChIP-qPCR. The box plot summarizes the chromatin enrichment of UTX and JMJD3 at each time point; $n=4,{ }^{*} P<0.05,{ }^{* *} P<$ 0.01. Mann-Whitney $U$ test in summary panels. c Aggregation plot of EZH1 near proximal promoters of all BD genes separated into stable, upregulated, and downregulated groups. d Inhibitory effect of EZH1 siRNA on bdDEGs activation as measured by RT-qPCR. EZH1 in HUVEC was suppressed by EZH1 siRNA and then treated with VEGFA for 1-4 h. EZH1 knockdown abolished the activation of six genes activated by VEGF. Plots show mean $\pm \mathrm{SD} ; n=4$, two-tailed Student's $t$-test: ${ }^{*} P<0.05$ compared to control at the same time point. e EZH1 upregulated bdDEG expression in HUVECs measured by RT-qPCR. Bar plots: mean \pm SD, two-tailed Student's $t$-test, $n=4,{ }^{* *} P<0.01,{ }^{* * *} P<0.001$.

activation of bdDEGs did not share the same mechanism of H3K27me3 demethylation as was observed during stem cell lineage commitment.

EZH1 recently has been shown to activate transcription of some genes, independent of H3K27me ${ }^{16,15,19}$. VEGF increased the EZH1 protein level at $1 \mathrm{~h}$ although there were no significant changes in its mRNA level (Supplementary Fig. 3a, b). Thus, we hypothesized that upregulation of bdDEGs might be due to the transcriptional activation driven by EZH1 at these regions, and performed ChIP-seq to assess the EZH1 dynamics upon VEGF treatment at four time points of $0,1,4$, and $12 \mathrm{~h}$. The results demonstrated EZH1 significantly increased its binding at 1 and $4 \mathrm{~h}$ after stimulation at proximity of both activate and stable but not downregulated BD genes (Fig. 3c). Comparing to late-regulated genes, early-upregulated genes have more discernible increasement at 1 and $4 \mathrm{~h}$ after VEGF treatment (Supplementary Fig. 2). This elevated relocation to chromatin at $1 \mathrm{~h}$ was recapitulated by ChIPquantitative polymerase chain reaction (qPCR) although not at $4 \mathrm{~h}$ (2.1-fold increase, $P=0.0046$; Supplementary Fig. 3c). Suppressor Of Zeste12 Protein Homolog (SUZ12), an essential scaffolding component of PRC2 reported to associated with EZH1, also relocated to activated $B D$ genes, which together with the evident of reduced EZH2 deposition suggested an atypical PRC2 complex mainly consisted of EZH1 and SUZ12, initially discovered within hematopoietic and heart cells, relocated to and elicited the bdDEG gene activation (Supplementary Fig. $3 \mathrm{c}-\mathrm{e})^{15,16}$.

To further interrogate if increased EZH1 relocation could in fact account for BD gene activation, we knocked down EZH1 with two independently designed $E Z H 1$ short interfering RNAs (siRNAs) and then treated with VEGF for up to $4 \mathrm{~h}$ (Supplementary Fig. 4a). Reversetranscription qPCR (RT-qPCR) demonstrated six tested $\mathrm{BD}$ genes selected from upregulated groups were activated by VEGF at $1 \mathrm{~h}$, but abrogated after transfection of either EZH1 siRNA (Fig. 3d). The inhibition also lasted to $4 \mathrm{~h}$ especially on IGFBP3 and ADAMTS1, the expression of which kept to be upregulated up to $4 \mathrm{~h}$. The ChIP-qPCR revealed the exist of $\mathrm{BD}$ at the same upregulated bdDEGs within human primary pulmonary artery (HPVECs), except PROX1, which is only expressed in venous and lymphatic endothelium (Supplementary Fig. 5a). We transfected the same EZH1 siRNA to HPVEC, which markedly suppressed the activation of all five tested bdDEG with similar inhibitory extent in HUVEC (Supplementary Fig. 5b). Reversely, overexpressing EZH1 in HUVEC cells increased the expression of six BD genes along with upregulated deposition of H3K27me1, a putative substrate of EZH1 methyltransferase (Supplementary Fig. 6a). In contrast, a reduced H3K27me3 deposition was also observed in EZH1 overexpressed cells, suggesting a decreased H3K27me3 demethylases (Fig. 3a, b) rather an increased EZH1, were responsible for VEGF-altered H3K27me3 deposition near active bdDEG (Supplementary Fig. 6b).

\section{bdDEG activation depended on EZH1-mediated RNAPII pausing release}

After assembling at the promoter, at many genes, RNAPII initiates transcription and enters early elongation, and then pauses at $30-50 \mathrm{bp}$ downstream of the TSS ${ }^{20}$. To complete the transcription, RNAPII need to be released from the pausing state. RNAPII pausing and pause release is rate-limiting for the transcription of many genes and is controlled by both positive and negative regulatory mechanisms $^{21}$. Our previous studies reveal that VEGFA promotes RNAPII pausing release, which is essential for VEGFA-stimulated transcription and angiogenesis ${ }^{11,20}$. Notably, EZH1 was reported to globally associate with H3K4me3 and release RNAPII pausing in myoblast cells $^{19}$. Therefore, we further hypothesized that the activation of bdDEGs was executed by EZH1 and EZH1mediated RNAPII pause release. To test this hypothesis, we first analyzed our previously published RNAPII ChIPseq data ${ }^{11}$. Metagene plots of RNAPII signal at upregulated bdDEGs illustrated that most of these genes had paused RNAPII (Figs. 1a and 4a, Supplementary Fig. 4). After $1 \mathrm{~h}$ of VEGFA treatment, more RNAPII entered into the gene body illustrating RNAPII pausing release ${ }^{20,22}$. The pausing index (PI: the ratio of RNAPII signal near the TSS compared to within the gene body, see Methods section) is a widely used indicator of the extent of RNAPII pausing. Consistent with the metagene plot, PI of the majority of upregulated bdDEGs decreased markedly at $1 \mathrm{~h}$ (Fig. 4b). 
A

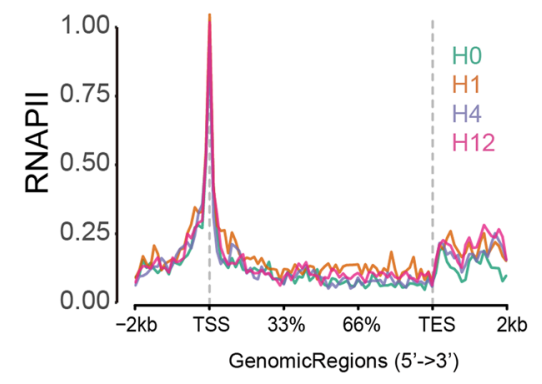

C
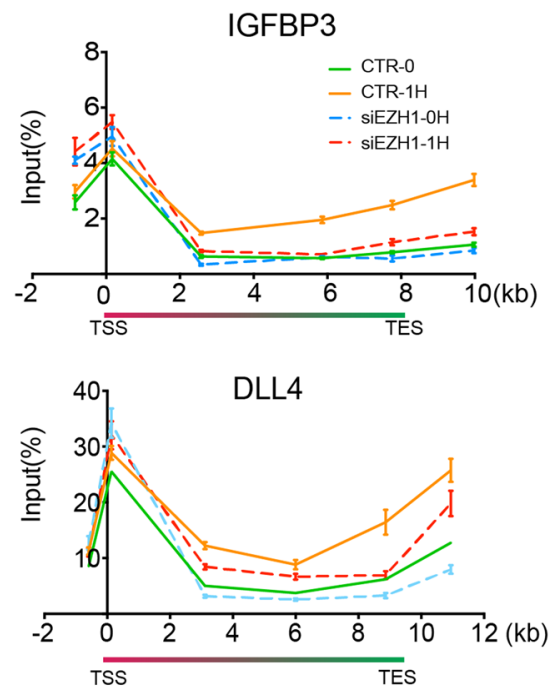

B
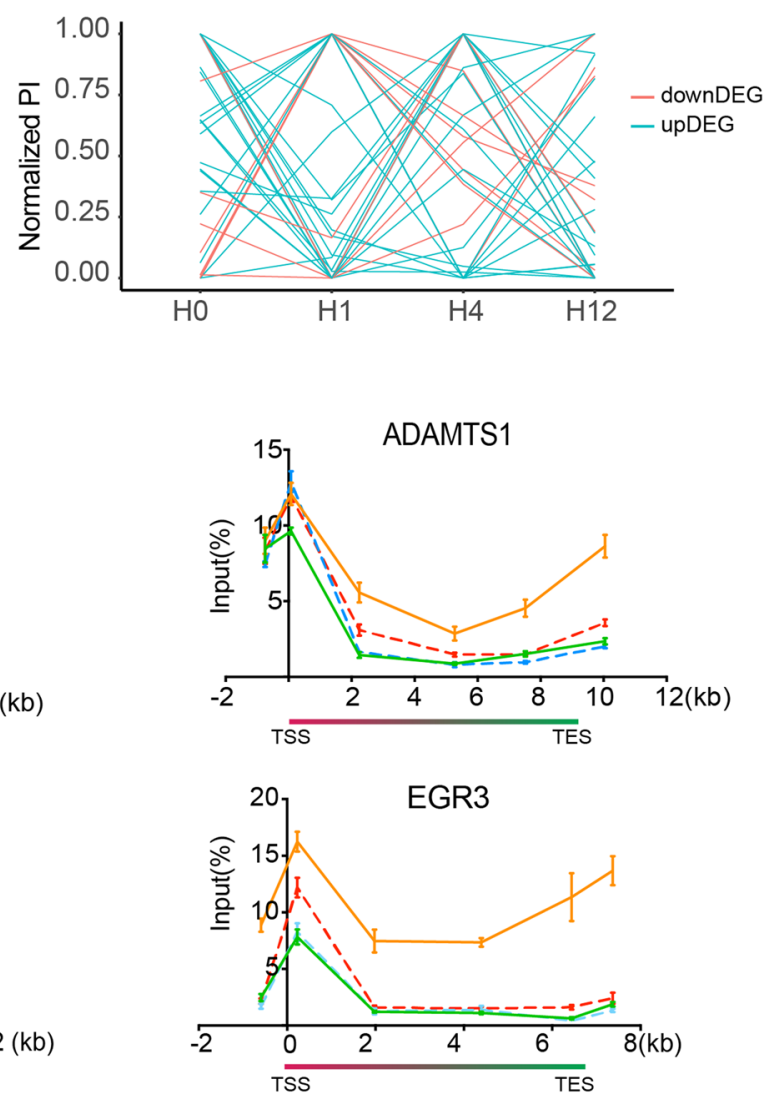

EGR3
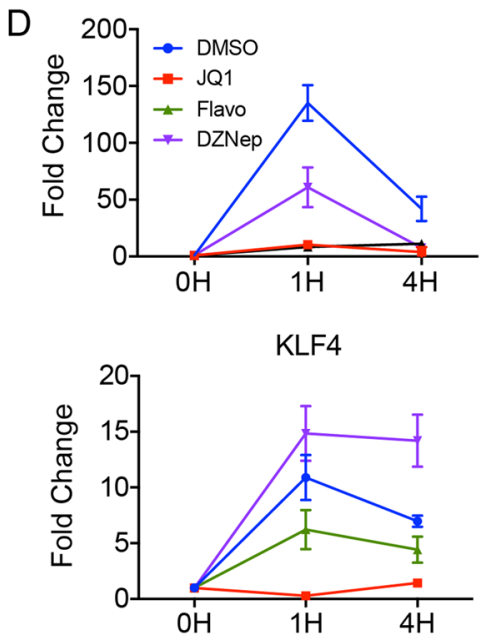

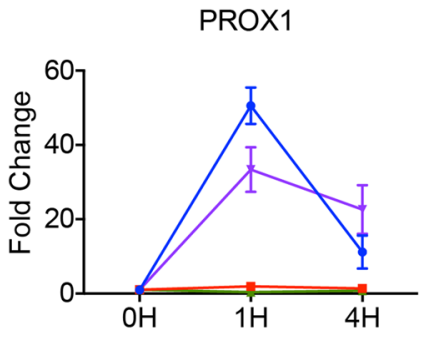

ADAMTS1

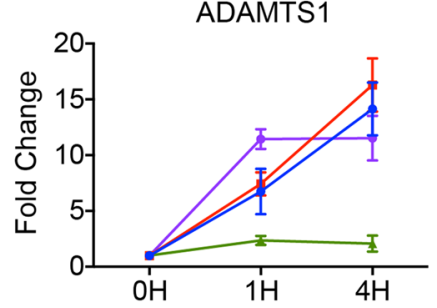

IGFBP3

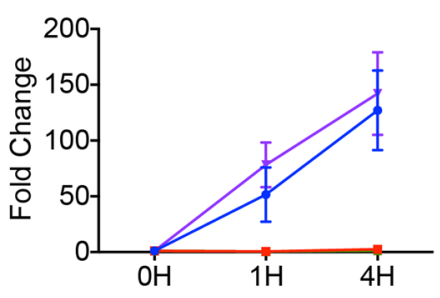

DLL4

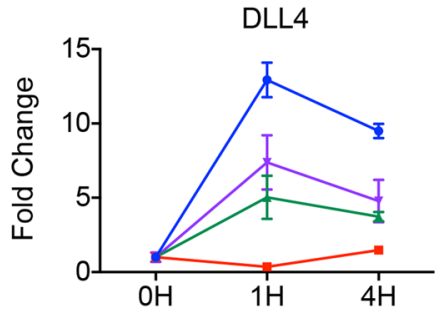

Fig. 4 EZH1-induced RNAPII pausing release was required for the activation of bdDEG. a Metagene plot showing RNAPII Occupancy of upregulated bdDEG at 0,1 , or $12 \mathrm{~h}$ of VEGFA treatment. VEGFA treatment stimulated release of paused RNAPII at $1 \mathrm{~h}$. $\mathbf{b}$ The RNAPII PI of downregulated bdDEG (red) and upregulated bdDEG (blue) at hour 0, 1, 4, and 12. c Effect of inhibition of RNAPII pausing release or PRC2 methyltransferase activity on VEGFA activation of bdDEGs, as measured by RT-qPCR. HUVECs were treated with VEGFA and/or small molecule inhibitors (JQ1, flavopiridol, and DZNep) or vehicle (DMSO). Inhibition of RNAPIl pause release (JQ1 and flavopiridol) but not PRC2 methyltransferase activity (DZNep) suppressed VEGFA-driven transcriptional activation of the six tested bdDEGs. Plots show mean \pm SD; $n=4$, two-tailed Student's $t$ test: ${ }^{*} P<0.05$ compared to control at the same time point. $\mathbf{d}$ RNAPII pausing status with or without $E Z H 1$ knockdown upon VEGF treatment for 0 or $1 \mathrm{~h}$, as measured by RNAPII ChiP-qPCR. EZH1 siRNA significantly abolished VEGF-induced RNAPII pausing release at four tested genes: IGFBP3, ADAMTS1, DLL4, and EGR3. Plots show mean $\pm \mathrm{SEM} ; n=4$. 
To further confirm that EZH1 mediated the RNAPII pausing release at the upregulated bdDEG, we compared the RNAPII signals measured by RNAPII ChIP-qPCR at multiples sites across the mRNA transcription regions of four tested genes before and after EZH1 knockdown. Suppression of EZH1 attenuated the induction of RNAPII occupancy at transcriptional and transcriptional termination regions of four tested genes, illustrating a disabled RNAPII pausing release caused by EZH1 depletion (Fig. 4c). Furthermore, to confirm the role of RNAPII pause release in bdDEG activation, we blocked pausing release with two inhibitors JQ1 and flavopiridol, which inhibit the RNAPII release proteins BRD4 and CDK9, respectively. At six tested upregulated bdDEGs, both JQ1 and flavopiridol significantly dampened VEGFA-induced activation, with the exception of ADATMTS1, which was not affected by JQ1 (Fig. 4d). In the same experimental context, DZNep, a general histone methyltransferase inhibitor, had a much weaker effect (Fig. 4d). Thus, these results indicated that VEGFA activation of bdDEGs depends on EZH1-enhanced RNAPII pause release, rather the loss of H3K27me3.

\section{$B D$ facilitated the silence of bdDEG though the recruitment of KDM5A}

DEGs with bivalent promoters were most highly enriched for genes that were rapidly upregulated $1 \mathrm{~h}$ after VEGFA and then rapidly downregulated (Fig. 1d, Supplementary Table 2). Mirroring this expression time course, H3K4me3 promoter occupancy at early upregulated bdDEGs declined notably after $1 \mathrm{~h}$ (Figs. 1e and 2a). We hypothesized that H3K27me3 at these early and transiently upregulated bdDEG promoters functions to accelerate erasure of $\mathrm{H} 3 \mathrm{~K} 4 \mathrm{me} 3$ and reduction of transcription. KDM5A, an H3K4me3 demethylase, was previously reported to physically interact with PRC2 (ref. ${ }^{6}$ ) and therefore was a candidate that might link H3K27me3 at bdDEGs to H3K4me3 removal. By ChIP-qPCR, KDM5A occupancy of these bdDEG promoters dramatically increased at $1 \mathrm{~h}$ (Fig. 5a). Furthermore, KDM5A but not $K D M 5 B$ siRNA suppressed the erasure of H3K4me3 and attenuated gene downregulation at $4 \mathrm{~h}$ (Fig. 5b, c, Supplementary Fig. 4a, b). Depletion of PRC2 by SUZ12 siRNA reduced KDM5A occupancy at these promoters (Fig. 5d and Supplementary Fig. 4a, b), consistent with PRC2-dependent recruitment of KDM5 $\mathrm{A}^{6}$. All these results suggest that H3K27me3 and KDM5A function together to accelerate the inactivation of rapidly upregulated bdDEGs by facilitating the removal of H3K4me3.

\section{BD governed endothelial cell migration and growth by modulating EGR3 expression}

The preferential regulation of VEGF responsive and angiogenic gene expression at bivalent regions lead us to hypothesize BD may function in regulating angiogenesis. We tested this hypothesis by upregulating EZH1 in HUVECs and examined its effect on endothelial cell migration, one of the symbolic features of angiogenesis. Upon VEGF stimulation, EZH1 overexpression enhanced endothelial cell migration $(P<0.001$, unpaired Student's $t$ test, Fig. 6a). EGR3, a well-defined proangiogenic factor reported to enhance endothelial migration and proliferation, was induced by VEGF over a 100-fold through an EZH-controlled RNAPII pausing release ${ }^{23,24}$ (Fig. 1d, Supplementary Table 2). Thus, we anticipated that EZH1induced HUVEC migration might be through the upregulation of EGR3. We knocked down EGR3 by EGR3specific siRNA that significantly attenuated EZH1's augmentation in migration (Fig. 6a, b, Supplementary Fig. 7a). Noteworthy, EZH1 also promoted the migration of HUVECs untreated with VEGF, which agreed with its function as a downstream effector of the VEGF pathway (Fig. 6a, b, Supplementary Fig. 7c, d). Conversely, knocking down EZH1 dampened VEGF-induced HUVEC cell migration, which was restored by EGR3 overexpression (Supplementary Figs. 6c, d and 7b). Depletion of KDM5A prolonged EGR3 expression (Fig. 5c). Consistently, KDM5A siRNA significantly increased the HUVECs' migratory ability (Supplementary Fig. 7a, b). Similar results were also observed in HPVECs, that HPVEC migration was promoted by EZH1 and suppressed by $K D M 5 A$, which were both $E G R 3$ dependent (Supplementary Fig. 7e-h).

Neovascularization requires extensive cell growt ${ }^{25}$. We first assessed the effects of EZH1 and KDM5A on the growth rate of HUVECs by measuring cell viability with CCK8 (Methods section). VEGF induced significantly increased cell growth after three days of administration (Fig. 6c). Knocking down EZH1 mildly but significantly attenuated this induction (Fig. 6c). Recovering EGR3 in the EZH1-depleted cells completely restored the growth of the HUVECs, which agreed with the pro-proliferation function of EGR3 shown by upregulating EGR3 alone ${ }^{24}$. Likewise, using KDM5A siRNA to extend EGR3 expression increased cellular proliferation (Fig. 6c). Conversely, ectopic expression of EZH1 enhanced VEGF-induced cell growth, which was abolished by further adding EGR3 siRNA (Supplementary Fig. 7i).

Angiogenic endothelial cells are able to form a network on the surface of extracellular matrix such as Matrigel, which is an indicator of angiogenic properties. We deployed the tube formation assay to evaluate the effects of EZH1, EGR3, and KDM5A on the endothelial network formation. EZH1 siRNA dampened the formation of HUVEC tubes as indicated by a shorter tube length within the measured regions (Fig. 6d, Supplementary Fig. 8a). Further upregulation of $E G R 3$ rescued the impaired tube formation capacity upon the depletion of EZH1 (Fig. 6d, 


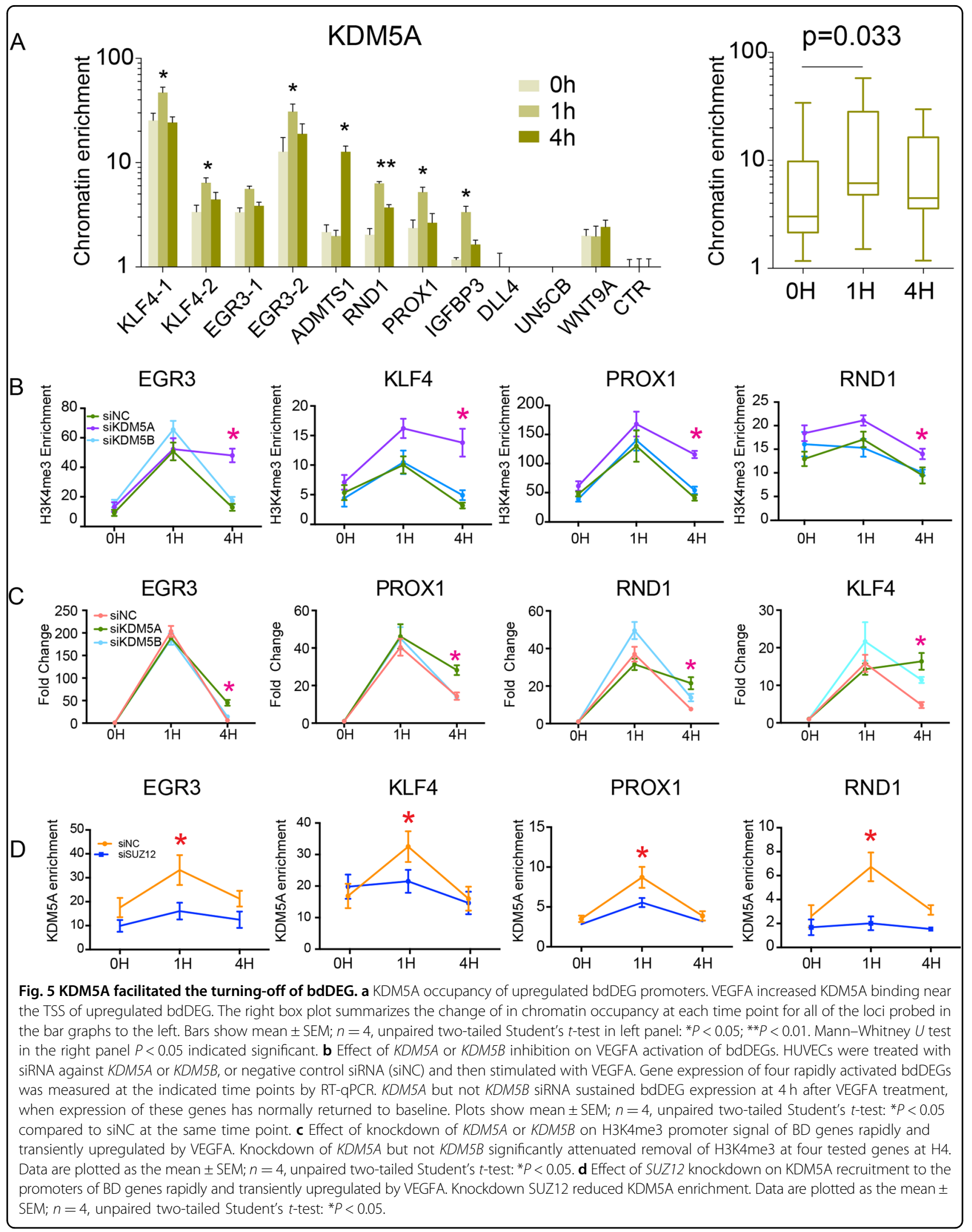


A

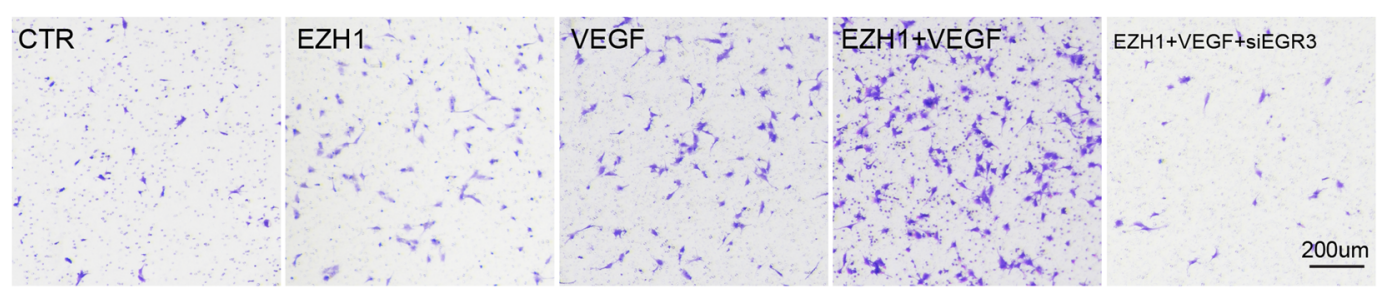

B

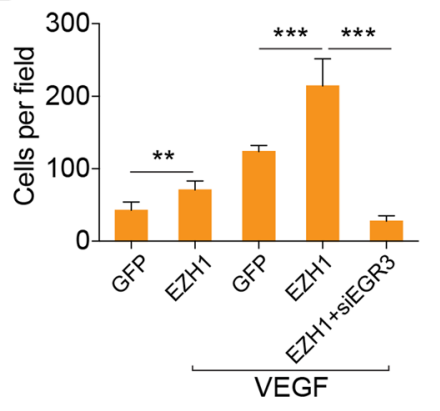

E

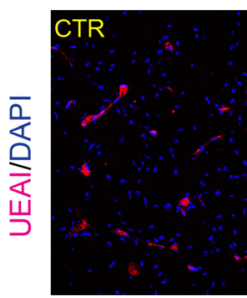

siEZH1+EGR3
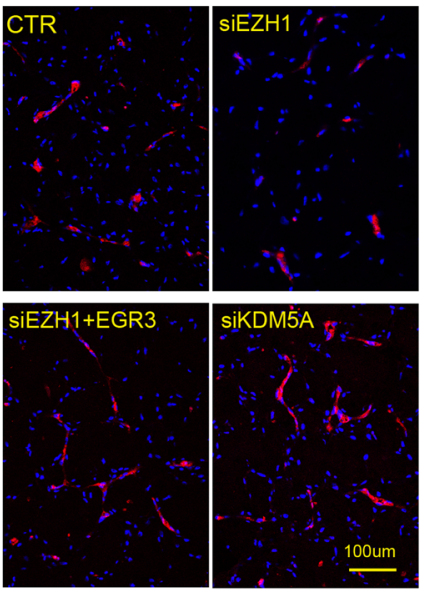

F

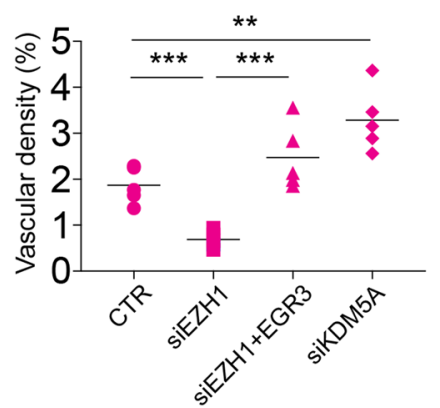

C

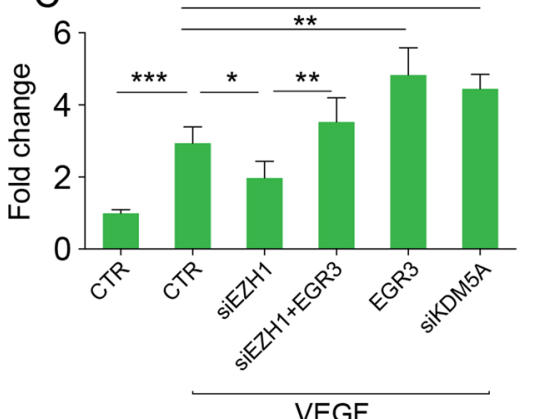

D

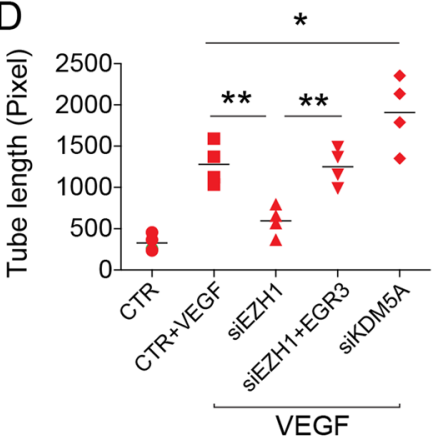

\section{G}

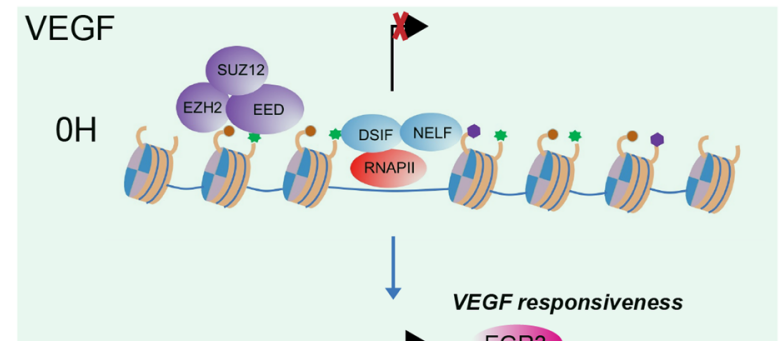

$1 \mathrm{H}$

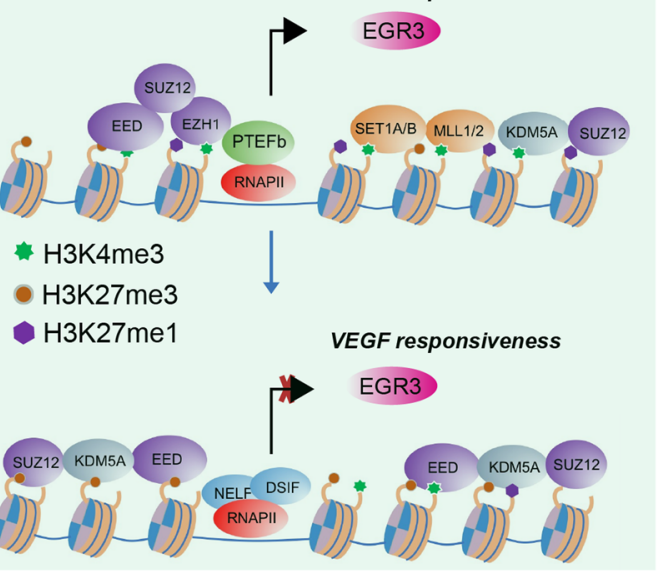

Fig. 6 The effects of EZH1, EGR3, and KDM5A on neovascularization. a, b Transwell assay showing EZH1 promoted HUVEC cell migration in culture conditions with or without VEGF, which was abolished by knocking down EGR3. a Migrated HUVECs on the transwell membrane revealed by crystal violet staining. $\mathbf{b}$ Calculation of migrated cells per field. Plots: mean $\pm S D,{ }^{*} P<0.01 ;{ }^{* *} P<0.001 ; n=6$, unpaired two-tailed Student's $t$-test. c Proliferation of HUVECs measured by the CCK8 method. The suppression of EZH1 abolished VEGF-induced HUVEC cell proliferation, which was reversed by addition of EGR3. Suppression of KDM5A enhanced cell proliferation. Bar plots: mean \pm SD; $n=5$, unpaired two-tailed Student's $t$-test, ns no significance, ${ }^{*} P<0.01 ;{ }^{* *} P<0.01$; ${ }^{* *} P<0.001$. d Tube formation of HUVECs on Matrigel coat. EZH1 siRNA dampened VEGF-induced tube formation of HUVECS, which was ameliorated by ectopic expression of EGR3; KDM5A suppression augmented VEGF-induced tube formation. Calculation of tube length in each tested group. Bar plots: mean $\pm \mathrm{SD}, n=4$, unpaired two-tailed Student's $t$-test, ${ }^{* *} P<0.01$, ${ }^{* * *} P<0.001$. e, f Matrigel assay evaluating the in vivo angiogenesis. e Representative images of neovasculature within transplanted Matrigel. UEAl-positive cells (magenta) indicating the wiring vasculature. $\mathbf{f}$ Dot plots of vascular density of UEAl-positive vasculature; mean $\pm S D, n=5$, unpaired two-tailed Student's $t$-test, ${ }^{* *} P<0.01,{ }^{* * *} P<0.001$. g Mechanistic model of BD's regulation of VEGFA responsiveness. 
Supplementary Fig. 8a). KDM5A siRNA augmented the tube formation of HUVECs primed with VEGF stimulation (Fig. 6d, Supplementary Fig. 8a). With a similar effect on endothelial cell proliferation and migration, ectopic expression of EZH1 promoted HUVEC tube formation, which depended on the upregulation of EGR3 (Supplementary Fig. 8b, c). Together, these lines of evidence demonstrate that the $\mathrm{BD}$ in endothelial cells controls VEGF-induced in vitro angiogenesis by modulating expression of its target gene EGR3.

\section{$B D$ governed in vivo angiogenesis}

To further probe the regulation of $\mathrm{BD}$ on in vivo angiogenesis, we carried out a Matrigel plug transplantation assay in immunodeficient mice (Methods section). Same as in the in vitro experiment, we first manipulated $E Z H 1, K D M 5 A$, and EGR3 levels in the HUVECs by using siRNA or lentivirus and then transplanted them to the rodent subcutis after mixing them with Matrigel and MSCs that supported the formation of functional vasculature. This assay demonstrated that suppression of $E Z H 1$ in endothelial cells impaired the neovascularization within the Matrigel, whereas suppression of $K D M 5 A$ enhanced it (Fig. 6e, f). Restoring the EGR3 in EZH1depleted cells also rescued their angiogenic capacity $(P<$ 0.001 , unpaired Student's $t$-test, Fig. 6e, f). A similar observation from both in vitro and in vivo angiogenesis assays concluded that the $\mathrm{BD}$ is able to control angiogenesis not only in vitro but in vivo.

\section{Discussion}

This study reveals a previously unrecognized role and mechanism of BDs in regulating signal-responsive genes. Before VEGFA stimulation of endothelial cells, EZH2containing PRC2 occupies bdDEGs and suppresses gene expression through RNAPII pausing. After VEGFA treatment, EZH1 rapidly replaces EZH2 and releases paused RNAPII with increasing H3K27me1 and H3K4me3 deposition possibly through the recruitment of H3K4me3 methyltransferases. Together, these actions rapidly increase gene transcription. On the other hand, the existence of $\mathrm{H} 3 \mathrm{~K} 27 \mathrm{me} 3$ at the $\mathrm{BD}$ facilitates later inactivation of early-responsive bdDEGs at 4 and $12 \mathrm{~h}$, H3K27me3 recruits KDM5A to these bdDEGs, resulting in removal of $\mathrm{H} 3 \mathrm{~K} 4 \mathrm{me} 3$ and transcriptional inactivation. The two faces of BD controlling the expression of VEGFresponsive genes, especially EGR3, play a pivotal role in the activation and inactivation of in vitro and in vivo angiogenesis (Fig. 6g).

\section{Bivalency remains at BDs in endothelial cells}

The functions of BDs have been well described during ES cell differentiation, but not clearly illustrated in the committed cells. Here, we revealed that bivalency bears dual roles in regulating VEGFA-induced gene expression but the interpretation of bivalency is entirely different. In the activation phase of VEGFA stimulation, rather to remove H3K27me3 for activation of gene expression, the BD was activated through a mechanism in which EZH1 released the paused RNAPII at these regions ${ }^{19}$. Although both $E Z H 1$ and $E Z H 2$ trimethylate histone $\mathrm{H} 3$ on lysine 27, EZH1 has been shown to positively regulate gene expression in multiple tissues. In myoblasts, EZH1 globally associated with H3K4me3 and RNAPII, promotes RNAPII elongation, and activates genes that govern the differentiation into skeletal muscle cells ${ }^{19}$. An EZH1/EZH2 switch mediated by GATA1/GATA2 transition was discovered in erythroid progenitor cells ${ }^{26}$. In the neonatal heart, EZH1 also positively regulated genes related to heart repair ${ }^{16}$. In line with these discoveries, we found in the blood vessels, EZH1 was redirected to the $\mathrm{BD}$ and positively regulated endothelial genes there in the context of VEGFA stimulation.

The bivalent nature of $\mathrm{BD}$ domains facilitated timely inactivation of VEGFA-upregulated genes: H3K27me3 at these domains was bound by PRC2, leading to KDM5A recruitment, H3K4me3 reduction, and gene silencing. Unlike the complete erasure of H3K4me3 during ES differentiation, the reduction of $\mathrm{H} 3 \mathrm{~K} 4 \mathrm{me} 3$ at endothelial cell $\mathrm{BDs}$ was incomplete, leaving the BD competent to properly respond to subsequent VEGFA stimulation. The mechanism that limits KDM5A demethylation of these $\mathrm{BDs}$ is not known and will be an interesting area for future exploration.

\section{Potential functions of BDs in adult tissue}

BDs are not restricted to multipotent stem cells; they are also found in differentiated, mature cells. Many tissues have been found to have over $1000 \mathrm{BDs}^{4}$. The function of BDs in mature cells has not been elucidated. Here, we showed that BDs in endothelial cells are able to regulate VEGFA-stimulated transcription and angiogenesis. This further emphasizes there are critical functions of BD in adult tissues. Clarifying BD's function and regulation in other tissues or other important biological processes will become an interesting and important area for future exploration.

\section{Materials and methods \\ Cell culture}

For routine culture, HUVECs (CC-2519, Lonza) and human pulmonary artery endothelial cell (HPAEC, CC2530, Lonza) between three to seven passages were maintained in endothelial growth medium medium 2 (EGM2, cc-3162, Lonza) with all the growth factor supplied. For VEGFA stimulation experiments, HUVECs were cultured overnight in basal endothelial cell growth medium 2 medium (EBM2, CC-4176 Lonza) with 1\% 
fetal bovine serum (FBS). A total amount of $50 \mathrm{ng} / \mathrm{ml}$ VEGFA was then added, and cells were collected at 0,1 , 4, and $12 \mathrm{~h}$ for ChIP-qPCR, ChIP-seq, RNA-seq, and RTqPCR. Where indicated, flavopiridol $(100 \mathrm{nM})$, JQ1 $(500 \mathrm{nM})$, or DZNep $(500 \mathrm{nM})$ were added $1 \mathrm{~h}$ before VEGFA stimulation.

Human MSCs (hMSCs) were purchased from Lonza (Catalog \#: PT-2501) and cultured in $\mathrm{MSCBM}^{\mathrm{TM}}$ MSC basal medium (PT-3238, Lonza).

siRNAs were synthesized at GenePharma with all sequences listed in the Supplementary Table 3. A total amount of $10 \mathrm{ng} / \mathrm{ml}$ siRNA were transfected with Lipofectamine $^{\circledast}$ RNAiMAX regent manufactured by Thermo Fisher Scientific. The serum starvation was performed one day after siRNA transfection.

\section{Mice}

All mice experiments were performed under protocols approved by the Institutional Animal Care and Use Committee of Shanghai Jiao Tong University.

\section{Matrigel plug assay}

Transplanted Matrigel plug assays were performed as described previously ${ }^{11}$. Briefly, $1 \times 10^{6}$ HUVECs at 4-6 passage and $2 \times 10^{6}$ human MSCs were mixed with $200 \mu \mathrm{l}$ ice cold Matrigel (Corning, 356237) and injected into the subcutis of 6-8-week-old male nude mice (Si Lai Ke Experimental LLC, China) that were randomly subjected to different treatments after excluding the unhealthy entities. Five mice were assigned for each group. The Matrigel plugs were dissected out and embedded in paraffin. Ulex Europaeus Agglutinin I (UEAI, 1:200, Vector Labs), a lectin that recognizes human but not mouse endothelial cells, was used to reveal the vascular network formed by the transplanted HUVECs. Images were acquired with a Nikon A1Si confocal microscope, and UEAI positive vasculature was measured using ImageJ.

\section{Western blot}

Total proteins were extracted from $2-5 \times 10^{6}$ HUVECs by using RIPA buffer (2.5 mM Tris-HCl PH7.4, $150 \mathrm{mM} \mathrm{NaCl}$, $1 \%$ NP40, $1 \%$ sodium dexycholate, $0.1 \%$ sodium dodecyl sulfate (SDS), and protease inhibitor cocktails). The protein concentration was measured with BCA Protein Assay (23225, Pierce). Equal amounts of protein were loaded onto each well, separated on 10\% SDS-polyacrylamide gel electrophoresis gel, and transferred to immobilon-P PVDF membrane (IPVH000010, Millipore). Primary antibodies (Supplementary Table 4) were used to specifically probe the tested proteins. The protein bands were visualized with Immobilon Western Chemiluminescent HRP substrate (WBKLS0500, Millipore) and imaged on Amersham Imager 600.

\section{RT-PCR}

A total of $1 \times 10^{6}$ HUVECs were collected with cell lysis buffer RLT with $1 \%$ of 2-mercaptoemethanol. Total RNA was purified using the RNeasy Mini Kit with on-column DNase digestion. RNA quantity and quality were assessed by NanoDrop Spectrophotometer (Thermo Fisher). RNA samples with A260/A280 ratio > 1.8 were saved for RTqPCR or mRNA-seq.

For RT-qPCR, first strand cDNA was synthesized from $1 \mu \mathrm{g}$ total RNA with the SuperScript III First-strand synthesis System (Thermo Fisher). RT-PCR tests were carried out with PowerUp SYBR ${ }^{\mathrm{TM}}$ Green Master Mix (Thermo Fisher) and detected by StepOnePlus Real-Time PCR systems (Thermo Fisher).

\section{ChIP-qPCR and ChIP-seq}

ChIP was performed as described previously with minor optimization under the ENCODE guideline ${ }^{27}$. Approximately $2-10 \times 10^{7}$ HUVECs after treatment with VEGFA for $0,1,4$, or $12 \mathrm{~h}$ were crosslinked with $1 \%$ formaldehyde for $10 \mathrm{~min}$ at room temperature. After neutralizing with $0.125 \mathrm{M}$ glycine for $5 \mathrm{~min}$, the nuclei were extracted with $5 \mathrm{ml}$ hypotonic buffer (20 mM HEPES pH 7.5, $10 \mathrm{mM}$ $\mathrm{KCl}, 1 \mathrm{mM}$ EDTA, $0.2 \%$ NP40, 10\% glycerol, $1 \times$ Protease Inhibitor Cocktail (PI; Roche), and then suspended in the sonication buffer $(20 \mathrm{mM}$ Tris $\mathrm{Cl} \mathrm{pH}$ 8.0, $2 \mathrm{mM}$ EDTA, $150 \mathrm{mM} \mathrm{NaCl}, 1 \% \mathrm{NP} 40,0.1 \%$ SDS, and $1 \times$ protease inhibitor). Chromatin were sheared by sonication (Bioruptor Plus, Diagenode: $30 \mathrm{~s}$ per cycle for 30 cycles). The sheared chromatin was precleared with $30 \mu \mathrm{l}$ protein $\mathrm{G}$ Dynabeads (ThermoFisher Scientific) for $1 \mathrm{~h}$, incubated with 5-10 $\mu$ g ChIP antibody (Supplementary Table 4) at $4{ }^{\circ} \mathrm{C}$ overnight and then pulled down by Protein G Dynabeads (ThermoFisher) for $4 \mathrm{~h}$ at $4{ }^{\circ} \mathrm{C}$. The beads with ChIPed DNA were rinsed three to five times with RIPA buffer (50 mM HEPES pH 8.0, 1 mM EDTA, 1\% NP40, $0.7 \%$ sodium deoxycholate, $1 \%$ TritonX-100, $0.5 \mathrm{M} \mathrm{LiCl}$, and $1 \times$ protease inhibitor) and then decrosslinked in SDS buffer $(50 \mathrm{mM}$ Tris- $\mathrm{Cl} \mathrm{pH} 8.0$, and $1 \% \mathrm{SDS})$ at $65^{\circ} \mathrm{C}$ for $12-16 \mathrm{~h}$. The reversely crosslinked DNA was purified with QIAquick PCR purification columns after digestion with RNase A and proteinase for $1 \mathrm{~h}$ each.

The purified DNA for ChIP-qPCR was directly detected with PowerUp SYBR ${ }^{\mathrm{TM}}$ Green Master Mix (Thermo Fisher). The primers for each tested site are listed in Supplementary Table 3. The chromatin occupancy was calculated with the following formula: chromatin occupancy $=2^{\wedge}[\triangle \mathrm{Ct}$ of test site (ChIP-input) $-\triangle \mathrm{Ct}$ of control site (ChIP-input)].

For ChIP-seq, ChIP DNA was converted to Illumina sequencing libraries using the NEBNext ${ }^{\circledast}$ ChIP-seq Library Prep Master Mix Set (NEB) by following the manufacturer's instructions. Size selection of DNA libraries after adding the sequencing adaptors was 
performed on agarose gels rather than with beads. The barcoding primers were custom prepared (Supplementary Table 3). The quality of the constructed libraries was evaluated by Tape station 2200 (Agilent). To obtain optimized cluster density in the flow cells, the barcoded DNA was quantified using the qPCR NGS library quantification kit (Illumina). Finally, 50 nt single-end reads were produced from Illumina HiSeq2000 or 2500 platforms.

\section{RNA-seq}

RNA-seq was performed as described ${ }^{11}$. In brief, $5 \mu \mathrm{g}$ total RNA from HUVECs was purified with the Dynabeads mRNA DIRECT Purification Kit (Thermo Fisher) following the manufacturer's instructions. Oligo-dT beads were applied twice to purify the polyadenylated RNA in order to minimize the rRNA contamination. The libraries were constructed using Scriptseq V2 RNA-Seq Library Preparation Kit (Illumina). We used custom primers in the last step of PCR to add barcodes to the library DNA. The Illumina HiSeq 2500 platform was deployed to yield $50 \mathrm{nt}$ pair-end reads. Two biological replicates at each time point of VEGFA treatment were sequenced.

\section{ChIP-seq}

Sequencing reads were aligned to human genome hg19 using Bowtie2 with the default option. Duplicates were marked by Picard. Low quality reads having a mapping quality $<15$ were abandoned. MACS2 was applied to call peaks from aligned BAM files in the default setting. Peaks falling into blacklist regions (https://sites.google.com/site/ anshulkundaje/projects/blacklists) were removed. To visualize histone signals at BD regions, the ngs.plot.r in ngsplot package (https://github.com/shenlab-sinai/ngsplot) was used (-G hg19-R genebody-L 5000-GO total-SC global).

\section{PI calculation}

PI was calculated as described ${ }^{12}$ based on the RNAPII ChIP-seq. The RNAPII signal was first normalized by an input signal at the same region. The ChIP signal at the TSS $(-50$ to $+300 \mathrm{bp})$ and gene body region $(-300$ of TSS to $+2 \mathrm{~kb}$ of transcriptional end site, TES) was calculated using a home-written software package of PIC (https://github.com/binglab-SJTU/PIC) and the formula: $\mathrm{PI}=($ normalized signal at TSS/length of TSS)/(normalized signal at gene body/length of gene body).

\section{K-means cluster}

ChIP-seq signals of each histone modification (H3K4me2, H3K4me3, H3K27ac, and H3K27me3) near the TSS (TSS $\pm 1 \mathrm{~kb}$ ) were calculated by the PIC package and then normalized to the signal of input. The number of clusters was estimated by the silhouette method and then grouped by $K$-means clustering. Fisher's exact test was used to evaluate the enrichment of DEGs and bdDEGs in each TSS cluster.

\section{RNA-seq analysis}

RNA-seq reads were aligned to human genome hg19 using Tophat2 (23). Cuffquant (24) was used to quantify the gene abundances with options '--multi-read-correct --frag-bias-correct'. Differential gene expression was assessed with CuffDiff (24) with options '--library-normmethod geometric --dispersion-method pooled --compatible-hits-norm --multi-read-correct --min-alignmentcount 10 --FDR 0.1 --frag-bias-correct'. Genes with fold change $\geq 2$ and FDR $\leq 0.1$ at hour 1,4 , or 12 in pairwise comparison to hour 0 were selected as DEGs. A total of 901 DEGs, including 37 polyadenylated IncRNAs, were used for analysis in this study.

\section{Tube formation assay}

The tube formation assay was performed as described ${ }^{28}$. Briefly, 24 well plates were coated with $300 \mu \mathrm{l}$ of ice-cold Matrigel (Corning, 356237, 1:2 dilution with $200 \mu \mathrm{l}$ EGM2 medium). After $1 \mathrm{~h}$ of gelatinization at $37^{\circ} \mathrm{C}$ within a cell culture incubator, $1-5 \times 10^{5}$ HUVECs treated with siRNA or infected with 1-3 MOI of lentivirus were plated on the gel surface with or without incubation of $30 \mathrm{ng} / \mathrm{ml}$ VEGFA. The endothelial tubes formed between $6-16 \mathrm{~h}$ and were imaged by a Nikon SMZ800N microscope. Total tube length was measured with ImageJ software from the acquired pictures.

\section{In vitro cell proliferation}

HUVECs were transfected with $10 \mathrm{~nm} / \mathrm{ml}$ of EZH1 or KDM5A siRNA or infected with EZH1 or EGR3 lentivirus (1-3 MOI) for $24 \mathrm{~h}$, reseeded to 96 well plates (1000-3000 cells/well), and treated with $30 \mathrm{ng} / \mathrm{ml}$ of VEGFA for $72 \mathrm{~h}$. The cells' viability was measured using a Cell Counting Kit-8 (CCK8, Beyotime \#C0042) following the manufacturer's instructions. The $\mathrm{OD}_{450}$ value was quantified with an ELISA reader (BioTek) and converted to cell numbers using a standard curve drawn with a series of increasing cell numbers.

\section{Transwell migration assay}

Transwell assays were carried out as previously described $^{28}$. HUVECs and HPVECs were transfected with EZH1, KDM5A, or EGR3 siRNA or infected with EZH1, EGR3 lentivirus (1-3 MOI) for $48 \mathrm{~h}$, trypsinized into single cells, and resuspended in migration medium (EBM2 $+1 \% \mathrm{FBS})$. Transwell chambers (Corning 3422, pore size $8 \mu \mathrm{m}$ ) were assembled in 24 well plates. Then, $1 \times 10^{5}$ HUVECs or HPVECs in $100 \mu \mathrm{l}$ of migration medium were added to the upper side of the transwell chamber, and $600 \mu \mathrm{l}$ of migration medium with or without $30 \mathrm{ng} / \mathrm{ml}$ VEGF was added to the lower well to induce migration. 


\section{Acknowledgements}

We gratefully thank Dr. Ruei-zeng Lin and Dr. Juan M. Melero-Martin for providing the training and protocol for the in vivo Matrigel plug assays. B.Z. is funded by the National Science Foundation of China (91539109, 31671503), A Thousand Young Talent Award (16Z127060017), Innovation Program of Shanghai Municipal Education Commission (2017-01-07-00-01-E0028), and The National Key Research and Development Program of China (2018YFC1312702, 2018YF(1002400).

\begin{abstract}
Author details
'Department of Pediatric Cardiology, Xin Hua Hospital, School of Medicine, Key Laboratory of Systems Biomedicine, Shanghai Center for Systems Biomedicine, Shanghai Jiao Tong University, Shanghai 200240, China. ${ }^{2}$ School of Life Science and Food Engineering, Huaiyin Institute of Technology, Huaian 223003, China. ${ }^{3}$ Department of Biostatistics and Computational Biology, Dana-Farber Cancer Institute and Harvard T.H. Chan School of Public Health, Boston, MA 02215, USA. ${ }^{4}$ Renji-Med Clinical Stem Cell Research Center, Renji Hospital, School of Biomedical Engineering, Shanghai Jiao Tong University, Shanghai 200127, China. ${ }^{5}$ Department of Cardiology, Boston Children's Hospital, Boston, MA 02115, USA. ${ }^{6}$ Harvard Stem Cell Institute, Cambridge, MA 02138, USA
\end{abstract}

\section{Author contributions}

B.Z. and J.H.C. conceived the project. X.D.L., S.S.Z., P.Y.Y., S.Y.W., H.J.Y., S.M.S, F.Z., Z.X.L., L.L., H.Y.L., Y.Z., and W.W.T. performed the experiments and analyzed the data. J.H.C. and S.P.G. performed computational analysis, J.H.C., G.C.Y., W.T.P., and B.Z. designed the study and wrote the manuscript. Y.Z. assisted with the proliferation assay and ChIP-seq. S.C., Y.W.C., and K.S. were involved in the study design and manuscript revision. G.C.Y., W.T.P., and B.Z. oversaw all data analysis.

\section{Data availabiility}

All raw and processed NGS data are available in GEO 109626 and 139077 with token of 'gfyduwmkhbqplop' for reviewer.

\section{Conflict of interest}

The authors declare that they have no conflict of interests.

\section{Publisher's note}

Springer Nature remains neutral with regard to jurisdictional claims in published maps and institutional affiliations.

Supplementary Information accompanies this paper at (https://doi.org/ 10.1038/s41419-020-2228-3)

Received: 2 April 2019 Revised: 31 December 2019 Accepted: 2 January 2020

Published online: 30 January 2020

\section{References}

1. Allis, C. D. \& Jenuwein, T. The molecular hallmarks of epigenetic control. Nat. Rev. Genet. 17, 487-500 (2016).

2. Rice, J. C. \& Allis, C. D. Histone methylation versus histone acetylation: new insights into epigenetic regulation. Curr. Opin. Cell Biol. 13, 263-273 (2001).

3. Bernstein, B. E. et al. A bivalent chromatin structure marks key developmental genes in embryonic stem cells. Cell 125, 315-326 (2006).
4. Voigt, P., Tee, W. W. \& Reinberg, D. A double take on bivalent promoters. Genes Dev. 27, 1318-1338 (2013).

5. Schuettengruber, B., Bourbon, H. M., Di Croce, L. \& Cavalli, G. Genome regulation by Polycomb and Trithorax: 70 years and counting. Cell 171, 34-57 (2017).

6. Pasini, D. et al. Coordinated regulation of transcriptional repression by the RBP2 H3K4 demethylase and Polycomb-Repressive Complex 2. Genes Dev. 22, 1345-1355 (2008).

7. Margueron, R. \& Reinberg, D. The Polycomb complex PRC2 and its mark in life. Nature 469, 343-349 (2011).

8. Lee, M. G. et al. Demethylation of H3K27 regulates polycomb recruitment and H2A ubiquitination. Science 318, 447-450 (2007).

9. Agger, $\mathrm{K}$. et al. UTX and JMJD3 are histone H3K27 demethylases involved in HOX gene regulation and development. Nature 449, 731-734 (2007).

10. Carmeliet, P. Manipulating angiogenesis in medicine. J. Intern. Med. 255 538-561 (2004)

11. Chen, J. et al. VEGF amplifies transcription through ETS1 acetylation to enable angiogenesis. Nat. Commun. 8, 383 (2017).

12. Hellstrom, M. et al. Dll4 signalling through Notch1 regulates formation of tip cells during angiogenesis. Nature 445, 776-780 (2007).

13. Seo, S. et al. The forkhead transcription factors, Foxc1 and Foxc2, are required for arterial specification and lymphatic sprouting during vascular development. Dev. Biol. 294, 458-470 (2006).

14. Cowan, C. E. et al. Kruppel-like factor-4 transcriptionally regulates VEcadherin expression and endothelial barrier function. Circ. Res. 107, 959-966 (2010)

15. Xu, J. et al. Developmental control of polycomb subunit composition by GATA factors mediates a switch to non-canonical functions. Mol. Cell 57, 304-316 (2015).

16. $\mathrm{Ai}, \mathrm{S}$. et al. Divergent requirements for $\mathrm{EZH1}$ in heart development versus regeneration. Circ. Res. 121, 106-112 (2017).

17. Gao, Z. et al. PCGF homologs, CBX proteins, and RYBP define functionally distinct PRC1 family complexes. Mol. Cell 45, 344-356 (2012).

18. Tavares, L. et al. RYBP-PRC1 complexes mediate H2A ubiquitylation at polycomb target sites independently of PRC2 and H3K27me3. Cell 148, 664-678 (2012).

19. Mousavi, K., Zare, H., Wang, A. H. \& Sartorelli, V. Polycomb protein Ezh1 promotes RNA polymerase II elongation. Mol. Cell 45, 255-262 (2012).

20. Day, D. S. et al. Comprehensive analysis of promoter-proximal RNA polymerase II pausing across mammalian cell types. Genome Biol. 17, 120 (2016).

21. Adelman, K. \& Lis, J. T. Promoter-proximal pausing of RNA polymerase ॥: emerging roles in metazoans. Nat. Rev. Genet. 13, 720-731 (2012).

22. Delmore, J. E. et al. BET bromodomain inhibition as a therapeutic strategy to target c-Myc. Cell 146, 904-917 (2011).

23. Liu, D., Evans, I., Britton, G. \& Zachary, I. The zinc-finger transcription factor, early growth response 3, mediates VEGF-induced angiogenesis. Oncogene 27, 2989-2998 (2008)

24. Suehiro, J., Hamakubo, T., Kodama, T., Aird, W. C. \& Minami, T. Vascular endothelial growth factor activation of endothelial cells is mediated by early growth response-3. Blood 115, 2520-2532 (2010).

25. Carmeliet, P. Angiogenesis in life, disease and medicine. Nature 438, 932-936 (2005).

26. Huang, J. et al. Dynamic control of enhancer repertoires drives lineage and stage-specific transcription during hematopoiesis. Dev. Cell 36, 9-23 (2016).

27. Zhang, B. et al. A dynamic H3K27ac signature identifies VEGFA-stimulated endothelial enhancers and requires EP300 activity. Genome Res. 23, 917-927 (2013).

28. Zhang, B. et al. Repulsive axon guidance molecule Slit3 is a novel angiogenic factor. Blood 114, 4300-4309 (2009). 